\title{
Distinct Functional and Structural Connectivity of the Human Hand-Knob Supported by Intraoperative Findings
}

\author{
Luciano Simone, ${ }^{1,3}$ Luca Viganò, ${ }^{2}$ Luca Fornia, ${ }^{1,3}$ Henrietta Howells, ${ }^{1,3}$ Antonella Leonetti, ${ }^{1,3}$ Guglielmo Puglisi, ${ }^{1,3}$ \\ Andrea Bellacicca, ${ }^{1,3}$ Lorenzo Bello, ${ }^{2}$ and Gabriella Cerri ${ }^{1,3}$ \\ ${ }^{1}$ Laboratory of Motor Control, Department of Medical Biotechnologies and Translational Medicine, Università degli Studi di Milano, Milan 20122, \\ Italy, ${ }^{2}$ Unit of Neurosurgical Oncology, Department of Oncology and Hemato-Oncology, Università degli Studi di Milano, Milan 20122, Italy, and \\ ${ }^{3}$ Humanitas Reasearch Hospital, Scientific Institute for Research, Hospitalization and Health Care (IRCCS), Milan 20089, Italy
}

Fine motor skills rely on the control of hand muscles exerted by a region of primary motor cortex (M1) that has been extensively investigated in monkeys. Although neuroimaging enables the exploration of this system also in humans, indirect measurements of brain activity prevent causal definitions of hand motor representations, which can be achieved using data obtained during brain mapping in tumor patients. High-frequency direct electrical stimulation delivered at rest (HF-DESRest) on the hand-knob region of the precentral gyrus has identified two sectors showing differences in cortical excitability. Using quantitative analysis of motor output elicited with HF DES-Rest, we characterized two sectors based on their excitability, higher in the posterior and lower in the anterior sector. We studied whether the different cortical excitability of these two regions reflected differences in functional connectivity (FC) and structural connectivity (SC). Using healthy adults from the Human Connectome Project (HCP), we computed FC and SC of the anterior and the posterior hand-knob sectors identified within a large cohort of patients. The comparison of FC of the two seeds showed that the anterior hand-knob, relative to the posterior hand-knob, showed stronger functional connections with a bilateral set of parietofrontal areas responsible for integrating perceptual and cognitive hand-related sensorimotor processes necessary for goal-related actions. This was reflected in different patterns of SC between the two sectors. Our results suggest that the human hand-knob is a functionally and structurally heterogeneous region organized along a motor-cognitive gradient.

Key words: cortical excitability; direct electrical stimulation; functional connectivity; hand-knob; structural connectivity

Significance Statement

The capability to perform complex manipulative tasks is one of the major characteristics of primates and relies on the fine control of hand muscles exerted by a highly specialized region of the precentral gyrus, often termed the "hand-knob" sector. Using intraoperative brain mapping, we identify two hand-knob sectors (posterior and anterior) characterized by differences in cortical excitability. Based on resting-state functional connectivity (FC) and tractography in healthy subjects, we show that posterior and anterior hand-knob sectors differ in their functional connectivity (FC) and structural connectivity (SC) with frontoparietal regions. Thus, anteroposterior differences in cortical excitability are paralleled by differences in FC and SC that likely reflect a motor (posterior) to cognitive (anterior) organization of this cortical region.

Received June 21, 2020; revised Jan. 2, 2021; accepted Jan. 10, 2021.

Author contributions: L.S., L.V., L.F., H.H., and G.C. designed research; L.S., L.V., L.F., H.H., A.L., G.P., and L.B. performed research; L.S., L.V., L.F., H.H., and A.B. analyzed data; L.S. wrote the paper.

Data were provided by the Human Connectome Project, WU-Minn Consortium (Principal Investigators: David Van Essen and Kamil Uğurbil; 1U54MH091657) funded by the 16 National Institutes of Health (NIH) Institutes and centers that support the NIH Blueprint for Neuroscience Research and by the McDonnell Center for Systems Neuroscience at Washington University.

L. Simone's present address: C'MoN, Cognition, Motion and Neuroscience Unit, Fondazione Istituto Italiano di Tecnologia, 16152 Genova.

We thank E. Borra for comments and suggestions.

The authors declare no competing financial interests.

Correspondence should be addressed to Luciano Simone at luciano.simone52@gmail.com.

https://doi.org/10.1523/JNEUROSCI.1574-20.2021

Copyright $\odot 2021$ the authors

\section{Introduction}

Human hand dexterity provides us with the means to perform skillful motor actions. Lesion studies show that human primary motor cortex (M1) is essential for producing dexterous hand movements (Porter and Lemon, 1993; Yousry et al., 1997). The hand representation of M1 has been radiologically termed the "hand-knob" region, referring to the region of precentral gyrus contained within the inverse $\Omega$-shaped orientation of the central sulcus visible on axial slices of magnetic resonance imaging (MRI; Yousry et al., 1997). This is usually referred as a single brain region, however cytoarchitectonic (Geyer et al., 1996) and electrophysiological studies (Crone et al., 1998) have argued that this area is heterogeneously organized, with much functional and anatomic diversity contained within it. 
Such diversity may bear similarities with the anatomo-functional features described by monkey studies (Strick and Preston, 1982; Rathelot and Strick, 2009). Indeed, monkey electrophysiological and neuroanatomical studies show multiple hand motor representations in $\mathrm{M} 1$ and premotor regions (Rizzolatti et al.,1988; Maranesi et al., 2012). While task functional MRI (fMRI)-based studies have contributed toward a better description of the functional complexities of the somatotopic organization of human M1 and premotor cortex (Binkofski et al., 1999; Meier et al., 2008), they usually fail to distinguish differences within the hand representations of motor cortex.

In the last decade, the development of awake intraoperative brain-mapping procedures for resection of brain tumors enabled the description of distinct hand regions with high spatial resolution (Fornia et al., 2018). Direct electrical stimulation (DES) applied to different sectors of human premotor cortex (PMd, $\mathrm{PMv}$ ) disrupts the execution of hand-object manipulation differently (Fornia et al., 2020), suggesting multiple hand representations related to different aspects of task control, supporting findings using non-invasive transcranial magnetic stimulation (TMS; Davare et al., 2006). Moreover, quantitative analysis of the motor output elicited with high-frequency DES delivered at rest (HF-DES-Rest) delivered on the hand-knob region has demonstrated differences in cortical excitability organized along anteroposterior direction, with higher excitability in posterior than anterior sectors (Viganò et al., 2019).

A similar anatomo-functional heterogeneity has been described also in the macaque. Within M1, Rathelot and Strick identified a posterior phylogenetically "new" subdivision from which cortico-motoneuronal projections originate, and an anterior phylogenetically "older" subdivision lacking these projections (Rathelot and Strick, 2009). By means of cortico-motoneuronal cells, the "new M1" acts directly on spinal motoneurons thus representing the crucial motor output for dexterous hand movements. The intraoperative neurophysiological setting offers the opportunity to collect more comparable data with those obtained in monkeys (Fornia et al., 2018, 2020; Rech et al., 2019), as opposed to non-invasive approaches using fMRI or TMS. Accordingly, the presence of two cortical sectors with different excitability in the human hand-knob suggests, despite their proximity, involvement in different circuits controlling different aspects of motor control. However, to our knowledge, the functional and structural networks underlying these hand representations remain undiscovered.

The development of MRI-based methods has made it possible to map functional connectivity (FC) and structural connectivity (SC) in the human brain. In the present study, we aim to investigate whether the difference in cortical excitability in the human hand-knob is attributable to two defined cortical sectors, a posterior and an anterior one (Viganò et al., 2019), also associated with characteristic functional and anatomic connectivity. Accordingly, we first define the posterior and anterior sectors of the hand-knob based on their cortical excitability, applying a quantitative analysis of the motor output elicited by delivery of HF-DES-Rest across the cortex. Seeds for connectivity analysis were defined using anatomic clustering methods [probability density function (PDF)] of stimulation sites defined within a large cohort of patients. Next, characteristic patterns of FC and SC of the two sectors were assessed using a cohort of healthy subjects provided by the WU-Minn Human Connectome Project (HCP) consortium (Van Essen et al., 2013). Healthy subject data were used to avoid possible biases caused by the presence of the tumor on the results.

\section{Materials and Methods}

Experimental design and statistical analyses

To investigate the FC and SC patterns of the human hand-knob, we first explored the electrophysiological features of the human hand-knob region in tumor patients. HF-DES-Rest was delivered on preserved precentral cortical sites of 46 patients (two left-handed; age ranging from 16 to 65 years, 24 female). Differences in cortical excitability were evaluated by comparing motor-evoked potentials (MEP) amplitude evoked by HFDES over two sites using a Mann-Whitney $U$ test. To compensate for sparse sampling, an estimation of anatomic clustering of the two cortical sectors was performed using PDF based on kernel density estimation (KDE), as previously published (Fornia et al., 2018; Puglisi et al., 2018). Finally, we used a normalization procedure to transform the results from native to standard space [Montreal Neurologic Institute (MNI)].

To avoid the effect of anatomic and functional reorganization of cerebral structures by the glioma on network definition of the hand-knob sectors, FC and SC were computed in healthy subjects. Although, the anatomic localization and the functional properties of the investigated motor sectors were preserved, the tumor could have promoted neuroplasticity processes, differently in each patient, of other cortical regions possibly functionally and structurally connected with the hand-knob sectors. We used 27 (age: 22-35, 16 females) healthy unrelated adults scanned as part of the HCP S1200 release of resting state fMRI (rsfMRI; Van Essen et al., 2013) to compute the FC of the anterior and posterior hand-knob. Seed-based analysis was performed by calculating the temporal correlation between the average blood oxygen level-dependent (BOLD) signals from a given hand-knob sector to all other voxels in the brain. Fisher $r$ to $\mathrm{Z}$ transformation was applied to correlation maps to achieve normality. Single subject connectivity maps were then entered into a second level general linear model to obtain population-level estimates. We used an uncorrected $p$ value height threshold of $<0.001$, with a cluster threshold of $p<0.05$ [cluster-size $p$-false discovery rate (FDR) corrected] as the extent threshold for the whole brain. Results were corrected for multiple comparisons FDR at seed-level $(p<0.05)$.

Significant differences in FC patterns between two hand-knob sectors was estimated by means of two paired $t$ tests for "between-source" differences [family-wise error (FEW); $p$-FWE $<0.05$ cluster-corrected, $p<0.05$ ].

\section{Patient selection}

The study included 46 patients (two left-handed; age ranging from 16 to 65 years) undergoing an asleep-asleep or an asleep-awake-asleep neurosurgical procedure with the aid of the brain mapping technique for removal of a tumor located in the right (26) or left (20) hemisphere. Some of patients with tumors located in the right hemisphere were included in the dataset of Viganò et al. (2019). Patients underwent the standard brain mapping procedure consisting in the HF-DES at $250 \mathrm{~Hz}$ (see Bello et al., 2014), aimed at the identification of the safe entry point and for preservation of cortical and subcortical structures responsive to the stimulation. Before the surgery, all patients were assessed for handedness, neurologic and neuropsychological evaluation of cognitive abilities (language, memory, executive function, and attention; for a comprehensive description of the neuropsychological battery, see Rossi et al., 2018; Puglisi et al., 2018, 2019), including upper limb apraxia (ideomotorapraxia test; see Spinnler and Tognoni, 1987). Only patients showing a score within the normal range on the entire neuropsychological battery were enrolled in the study. Further inclusion criteria were that the tumor was not infiltrating the investigated areas, no previous neurosurgical treatments. Only those patients with a short seizure history well controlled with only one anti-epileptic drug were included. All patients gave written informed consent to the surgical and mapping procedure, which followed the principles outlined in the World Medical Association Declaration of Helsinki of 1975, as revised in 2008, research involving human subjects. The study was performed with strict adherence to the routine procedure normally used for surgical tumor removal.

Intraoperative electrophysiological identification of the anterior and posterior hand-knob sector

In order to investigate the cortical excitability of the anterior and posterior precentral hand-knob region, HF-DES was delivered through a 
constant current monopolar stimulator (straight tip, $1.5 \mathrm{~mm}$ in diameter (Inomed), with reference/ground on the skull overlying the central sulcus) in train $(250 \mathrm{~Hz})$ of one to five pulses [pulse duration: $5 \mathrm{~ms}$; interstimulus interval (ISI): $3-4 \mathrm{~ms}$ ] at a repetition rate of $1 \mathrm{~Hz}$ and at variable intensity.

To avoid facilitation because of accidental voluntary movements, each patient was trained during the procedure to stay relaxed as possible with all body parts at rest. During the procedure, the central and precentral sulci were used as anatomic landmarks for identifying, respectively, the posterior and anterior sector of the hand-knob region.

The posterior sector was identified in the most posterior part of the $\Omega$ of the precentral gyrus while the anterior spot was localized at same mediolateral of the posterior one in proximity of the precentral sulcus. Proximity to the central or precentral sulci was defined by the morphology of the hand-knob area exposed under the surgical flap and always confirmed intraoperatively using the neuronavigation system (Brainlab).

First, the procedure consisted in identifying the motor threshold (MT) in the posterior hand-knob, i.e., the caudal MT (cMT). The cMT was defined as the minimum intensity (with fixed number of pulses) required to evoke stable (in amplitude) and highly repeatable (in time) MEPs from at least one hand-related muscle.

HF-DES-Rest was first delivered on the posterior sector at $10 \mathrm{~mA}$ with train of five pulses. If MEPs were evoked, the intensity of current was reduced in step of $1 \mathrm{~mA}$, until finding the minimum intensity needed to evoke reliable MEPs. For clinical purposes, the surgeon may need to decrease the number of stimuli (train of one or two pulses) and then adjust the intensity of current to find cMT parameters. Conversely, if $10 \mathrm{~mA}$ with five pulses were not effective in eliciting a motor output, the intensity was increased in step of $1 \mathrm{~mA}$, finally obtaining cMT parameters.

Once the cMT was identified, HF-DES at cMT parameters (same number of pulses and same current intensity delivered on the posterior sector) was delivered anteriorly at same mediolateral of the posterior site thus allowing the comparison of cortical excitability of the two sectors at single patient level. Number of stimulation trials and HF-DES parameters were constrained by clinical needs and no additional sites others than those required by the procedure were added to the brain mapping procedure. Establishing cMT and its cortical localization within handknob sector is crucial to select the most excitable sector corresponding with the densest cortico-motoneuronal sector for monitoring integrity of corticofugal pathways during surgery. An electrode was placed in this sector from a strip of four to eight contacts delivering HF-DES in trains of five pulses at $1 \mathrm{~Hz}$ during tumor resection to continuously monitor amplitude of hand-related MEPs.

The intraoperative time constrains because of the clinical context, prevented this mapping in eight patients, and in these cases, the cortical excitability of the two hand-knob sectors was evaluated through a different procedure. The posterior and anterior sectors were stimulated with the same number of pulses and intensity, thus by using identical stimulation parameters, which allowed a reliable elicitation of MEPs at both sites. Despite this procedure avoids performing a descending intensity curve to assess the cMT (see Table 1), the difference in cortical excitability could be evaluated by comparing the MEP amplitude evoked by HFDES over two sites (see below).

\section{Analysis of MEPs}

In compliance with the clinical procedure the electromyographic (EMG) signals of hand/forearm muscles were acquired by means of Inomed software (ISIS, Inomed, sampling rate $20 \mathrm{kHz}$, notch filter at $50 \mathrm{~Hz}$ ). The MEPs recorded during the procedure, were extracted from the acquisition system and resampled at $4 \mathrm{kHz}$ and analyzed offline by means of dedicated MATLAB software. For each trial, a time window of $100 \mathrm{~ms}$ succeeding stimulus delivery was considered. The mean EMG baseline activity and its $\mathrm{SD}( \pm 1 \mathrm{SD})$ were calculated from the last $25 \mathrm{~ms}$ of the time window (75-100 ms). Only MEPs whose EMG signal exceeded the average background \pm 1 SD were considered for analysis (Fornia et al., 2018). We analyzed the MEP evoked on forearm-hand muscles [i.e., extensor digitorum communis (EDC), first dorsal interosseous (FDI), abductor pollicis brevis (APB), abductor digiti minimi (ADM), and flexor carpi radialis (FCR)]. MEPs were evoked when the patients were awake or asleep and laid with all body parts at rest, avoiding any "facilitation" effect (i.e., increase of excitability) because of unforeseen upper limb movements.

Only those muscles responsive to at least five HF-DES-Rest trials in both areas were included in the statistical analysis. All the MEPs evoked in the different muscles of the forearm and hand were grouped by sector (posterior or anterior hand-knob). All MEPs amplitude values were standardized within the area and between muscles ( $z$ score). MannWhitney $U$ tests were used to assess statistical differences between the MEP amplitude elicited in the two different hand-knob subsectors.

\section{Definition of seed regions of interest}

Each stimulation site in the hand-knob in the left and right hemisphere was used to calculate a three-dimensional visualization of the most probable region where DES was effective in evoking MEPs. The stimulation sites were assigned to the anterior or to the posterior hand-knob based on the anatomic location (Fig. $1 A, B$ ), the lower current threshold eliciting a motor output and, on the occurrence, and amplitude of the MEP (see above). Within each patient, MNI coordinates of each stimulation site in the hand-knob were calculated as follows.

The postcontrast T1-weighted image (TR/TE 2000/10 ms; FOV 230 $\mathrm{mm}$; 176 slices; matrix, $400 \times 512$; SENSE factor 1.5 ) of each patient (the same loaded into the neuronavigation system during surgery) was used to perform the cortical surface extraction and surface volume registration computed with the FreeSurfer Software (Fischl, 2012). Subsequently, by means of Brainstorm (http://neuroimage.usc.edu/ brainstorm), the site coordinates were marked on the patient's 3D MRI native space. Then, the MRI and stimulation sites were coregistered to MNI space through unified segmentation implemented in Statistical Parametric Mapping 12 software (SPM12; https://www.fil.ion.ucl.ac.uk/ $\mathrm{spm} /$ ). The coordinates of each site of all patients were defined on the ICBM152 template to create a 3D reconstruction of both hemispheres. To avoid mismatch between native and MNI space possibly introduced by normalization steps, the results of the coregistration step (matching the native and MNI localization of the anterior and posterior commissures, median line, and ventricles) were checked in each patient. Finally, we visually inspected the location of the stimulated sites on the MNI template with respect to its localization in native space. All the sites (posterior and anterior hand-knob) matched with the original/native anatomic localization.

Each stimulation site assigned to the anterior or to posterior handknob was then used to calculate a three-dimensional visualization of the most probable anterior and posterior hand-knob sectors, respectively, based on the geodesic distance between stimulation sites where cMT was effective or not in evoking reliable MEP. To this aim, we performed a $\mathrm{PDF}$ based on KDE. Usually, the KDE is calculated considering the Euclidean distance between the points. This is not the case, since the stimulation points lay on a surface (the brain surface) and the Euclidean distance does not represent the actual distance between two points. To overcome this limit that can introduce erroneous approximations, we defined the distance as the shortest path laying on the brain surface between two points. Then we implemented the classical KDE as defined in literature (Silverman, 1986; Scott, 2015).

\section{rsfMRI acquisition}

FC of minimally preprocessed rsfMRI data of 27 healthy unrelated adults (age: 22-35, 16 females) from the HCP S1200 release of rsfMRI (Van Essen et al., 2013) were employed in this study. Data included 1200 frames of multiband, echoplanar imaging (Smith et al., 2013) acquired in $\sim 15 \mathrm{~min}$ with the following parameters: echo time, $33.1 \mathrm{~ms}$; flip angle, $52^{\circ}$; field of view, $280 \times 180 \mathrm{~mm}$; matrix, $140 \times 90$; and voxel dimensions, 2-mm isotropic resolution; TR, $0.72 \mathrm{~s}$ (Uğurbil et al., 2013). In this study, we analyzed one out of the four runs acquired for each subject (left-right encoded).

\section{rsfMRI data analysis}

The spontaneous fluctuations in BOLD signal of the anterior and posterior hand-knob seeds identified by DES were cross correlated voxelwise 
Table 1. Demographic, anatomic, and neurophysiological information

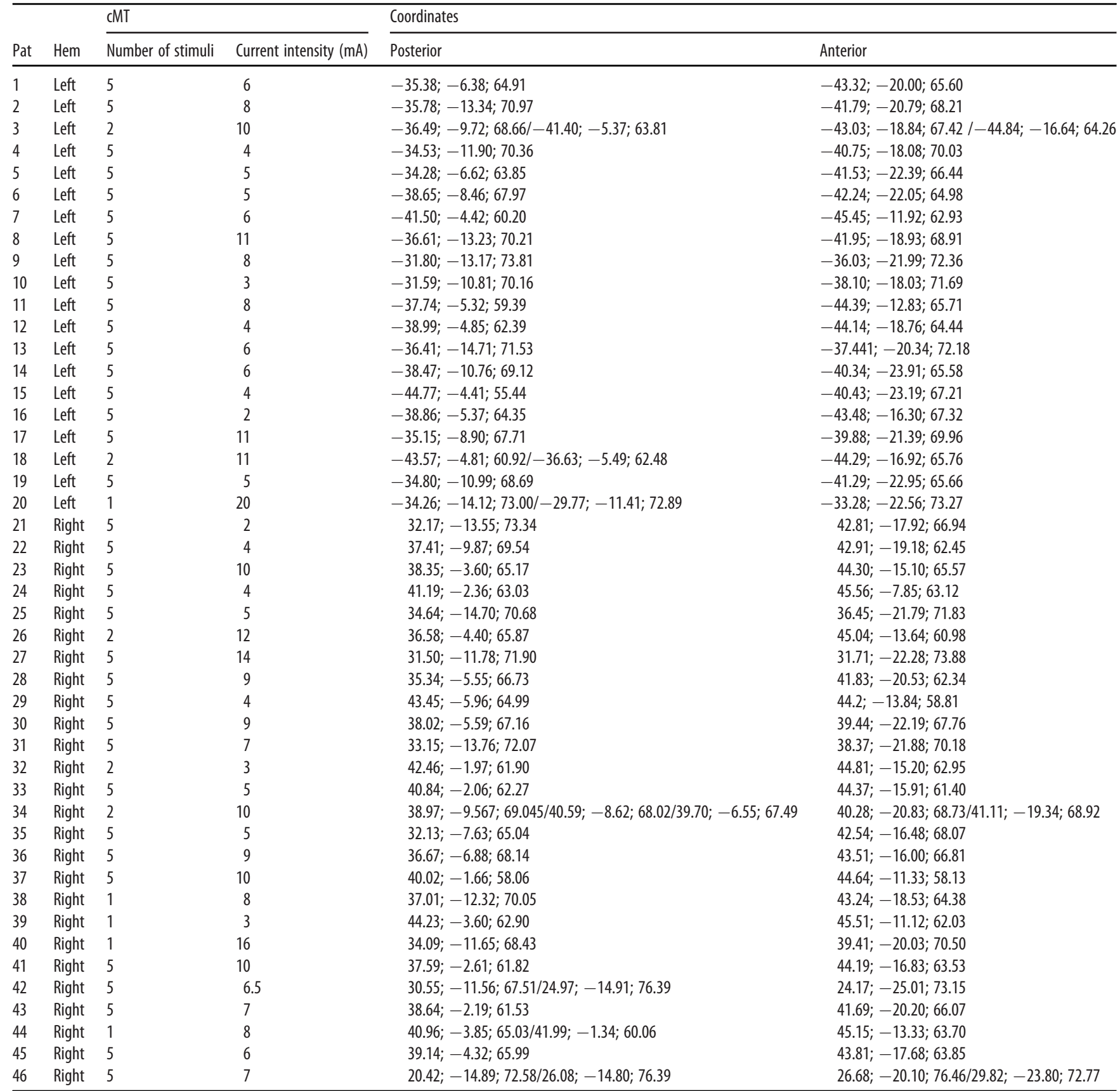

with every other signal time course in a whole brain analysis. This analysis generated whole-brain FC maps representing the strength of FC between each seed volume and the rest of the brain. Seeds-based correlational analyses were conducted using the FC (CONN) toolbox (https:// www.nitrc.org/projects/conn), a MATLAB/SPM-based cross-platform open-source software. We applied the minimal preprocessing pipeline for fMRI, developed by the HCP (Glasser et al., 2013), to attain spatial artifact/distortion removal, crossmodal registration, and alignment to standard space. Image preprocessing methods were performed by means of SPM12. Briefly, images were corrected for slice time and motion, coregistered with a high-resolution anatomic scan, normalized into the MNI space, resampled at $2 \mathrm{~mm}^{3}$, and smoothed with a Gaussian kernel of 6-mm $\mathrm{m}^{3}$ full-width half-maximum (Friston et al., 1995). After preprocessing, images were bandpass filtered to $0.008-0.09 \mathrm{~Hz}$ and motion regressed to diminish the impact of noise. Seed-based analysis was performed by calculating the temporal correlation between the average BOLD signals from a given seed to all other voxels in the brain. The data were then normalized by applying Fisher $\mathrm{r}$ to $\mathrm{Z}$ transformation. Seedbased FC was calculated for all subjects using a general linear model for Pearson's correlation coefficients estimation between the seed time course and the time course of all other voxels (first level analysis). The single subject connectivity maps were then entered into a second level general linear model to obtain population-level estimates. We used an uncorrected $p$ value height threshold of $<0.001$, with a cluster threshold of $p<0.05$ (cluster-size $p$-FWE corrected) as the extent threshold for the whole brain (second level analysis). Furthermore, we estimated the significant differences in FC patterns between two different seeds by means of two paired $t$ tests for between-source differences (FWE $p$-FWE $<0.05$ cluster-corrected, $p<0.05$ ), All group-level results were corrected for multiple comparisons (FDR).

\section{Analysis of structural connections}

The sectors identified from intraoperative mapping were compared with a population average tractography template (HCP-1021), produced from 

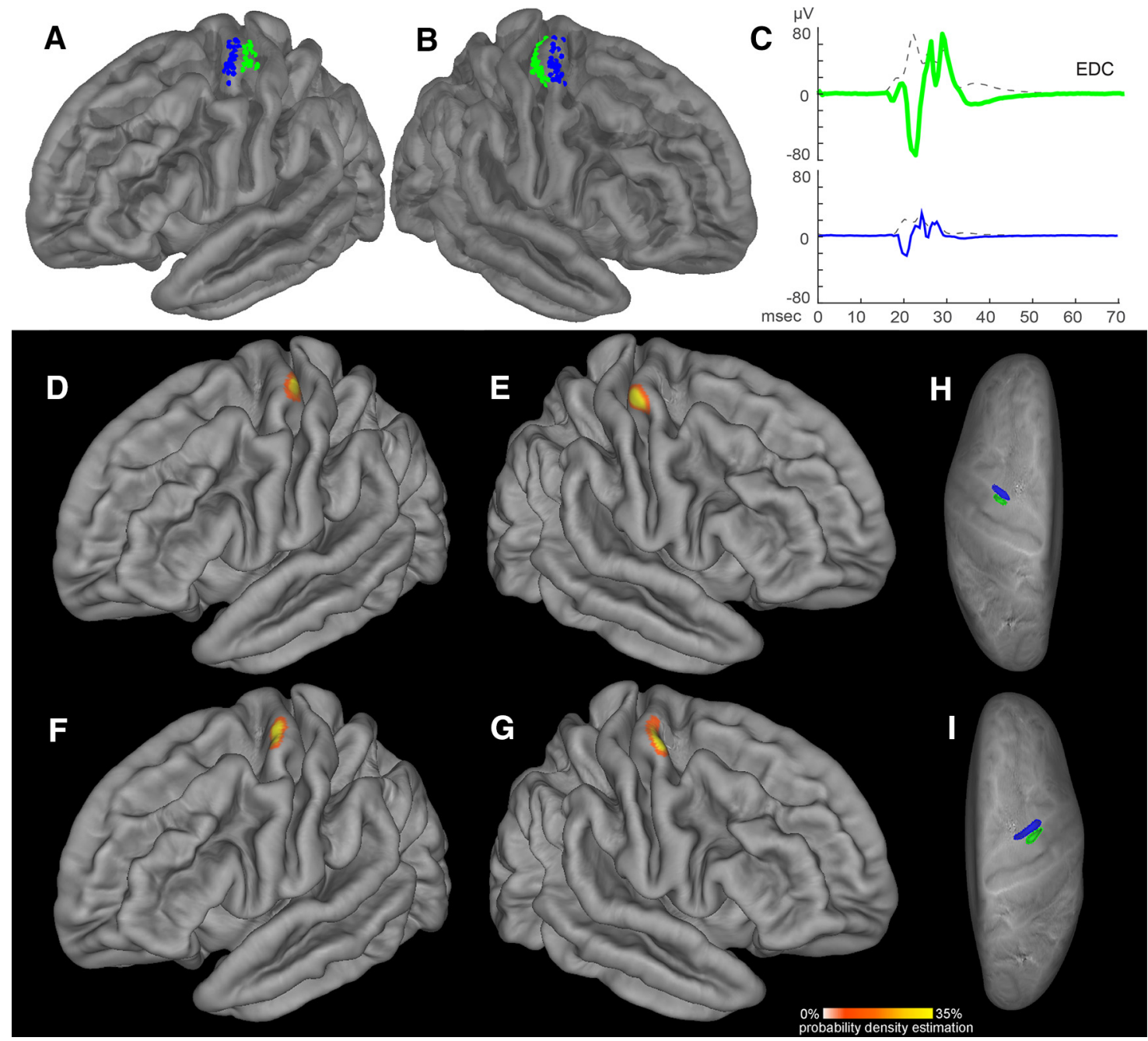

Figure 1. Anterior and posterior hand-knob seeds. Top panel, Distribution of the stimulation sites that were attributed to the anterior (blue) and posterior (green) hand-knob of the left ( $\boldsymbol{A}$ ) and right hemisphere $(\boldsymbol{B})$. $\boldsymbol{C}$, Representative EDC muscle MEP with higher amplitude evoked in the posterior hand-knob (green) and with lower amplitude evoked in the anterior hand-knob (blue) using the same stimulation parameters (number of pulses and intensity). Bottom panel, Posterior $(\boldsymbol{D}, \boldsymbol{E})$ and anterior $(\boldsymbol{F}, \boldsymbol{G})$ hand-knob seeds were estimated by means of a PDF. The left $(\boldsymbol{H})$ and right $(\boldsymbol{I})$ anterior (blue) and posterior (green) hand-knob sites projected on an inflated brain anatomic template using Caret software (version 5.65).

a total of 1021 subjects (Q1-Q4 1200 release, 2017), made available by Yeh et al. (2018). These data were acquired using a multishell scheme (b values 1000,2000 , and $3000 \mathrm{~s} / \mathrm{mm}^{2}$ ), with 90 sampling directions for each. In-plane resolution was $1.25 \mathrm{~mm}$, and slice thickness was $1.25 \mathrm{~mm}$. Diffusion data were reconstructed in MNI space using q-space diffeomorphic reconstruction (Yeh et al., 2010; Yeh and Tseng, 2011). A diffusion sampling length ratio of 2.5 was used with an output resolution of 1 $\mathrm{mm}$ and DSI Studio was used for visualizations. Sectors were registered using FSL tools and white matter tracts were defined projecting from these regions by a white matter anatomist (H.H).

\section{Results}

\section{Electrophysiological results}

A total of 103 stimulation sites were recorded (44 in the left and 59 in the right hemisphere), 49 of them were located in the posterior hand-knob (28 in the right hemisphere, patient mean 1, SD 0.3 ; 21 in the left hemisphere, patient mean 1, SD 0.2), while 54 were identified in the anterior hand-knob (31 in the right hemisphere, patient mean 1.2, SD 0.5; 23 in the left hemisphere, patient mean 1.1, SD 0.3).

Based on the cMT, differences in cortical excitability of the two hand-knob sectors were established in 38 patients (20 left and 18 right hemispheres; Fig. $1 A, B$ ). In these patients and in both hemispheres, DES delivered at cMT was effective in eliciting reliable MEPs in the posterior sector in at least one recorded muscle, while when applied on the anterior sector it systematically failed to evoke MEPs distinguishable from EMG background (Fig. 1C). The cMT corresponded to mean intensity of stimulation of $6 \mathrm{~mA}(2.5 \mathrm{SD})$ in left hemisphere and $6.9 \mathrm{~mA}(3.3$ $\mathrm{SD})$ in right hemisphere) with five pulses. When using only two pulses cMT corresponded to $10.5 \mathrm{~mA}(0.7 \mathrm{SD})$ in left hemisphere and $8.3 \mathrm{~mA}(4.7 \mathrm{SD})$ in right hemisphere. Stimulation at one pulse was delivered only in two patients (one left hemisphere, $\mathrm{cMT}=20 \mathrm{~mA}$; one right hemisphere, $c M T=8 \mathrm{~mA}$ ). In the remaining patients (eight right hemisphere) direct comparison of the effect of stimulation at cMT over the two subsectors was not performed for clinical constraints, in that it was not possible to assess the cMT. In these cases, MEPs were elicited on both sides (posterior and anterior) using the same stimulation parameters (i.e., number of pulses and intensity), thus the amplitude of MEPs was chosen as parameter for comparison of their excitability. MEP amplitudes evoked in the posterior hand-knob were significantly greater compared with MEP amplitudes evoked on anterior hand-knob $(p<0.1)$ thus confirming a higher excitability of the posterior hand-knob compared with the anterior. For further details, see Table 1. 
Table 2. Mean coordinated, relative SD, and number of voxels of each seed

\begin{tabular}{lccccccc}
\hline \multirow{3}{*}{ Seeds } & \multicolumn{3}{l}{ MNI coordinates } & & & \multirow{2}{*}{ Number of voxels } \\
\cline { 2 - 7 } & $\mathrm{x}$ & $\mathrm{y}$ & $\mathrm{z}$ & $\mathrm{sdx}$ & $\mathrm{sdy}$ & $\mathrm{sdz}$ & \\
\hline Left anterior hand-knob & -37 & -9 & 67 & 3.6 & 3.4 & 4.8 & 68 \\
Left posterior hand-knob & -42 & -20 & 67 & 3.0 & 3.2 & 2.8 & 48 \\
Right anterior hand-knob & 38 & -7 & 66 & 5.4 & 4.6 & 4.4 & 77 \\
Right posterior hand-knob & 42 & -18 & 66 & 5.7 & 4 & 4.6 & 61 \\
\hline
\end{tabular}

\section{Spatial localization of anterior and posterior hand-knob} seeds for rsfMRI

The probability density estimation was applied on the anatomic coordinates of each cortical site to form anatomically and functionally defined seeds corresponding to posterior or anterior hand-knob. The coordinates of each site were assigned to the anterior or to the posterior hand-knob according to the anatomic location, the current threshold eliciting a motor output, the occurrence and the amplitude of the evoked MEP. The surface volume obtained from each density estimation analysis was thresholded to include only those volumes showing a probability $\geq 35 \%$.

In each hemisphere, we identified two intraoperative seeds: anterior hand-knob (Fig. 1F,G) and posterior hand-knob (Fig. $1 D, E)$ both located in the precentral gyri. Figure $1 H, I$ shows, on the inflated brain, the anterior (blue) and posterior (green) hand-knob seeds of the left and right hemisphere, respectively. These seeds were then used to compute seed to voxel fMRIderived FC in healthy brains. Mean coordinated with relative standard deviation and number of voxels of each seed were reported in Table 2 .

\section{Functional networks of anterior and posterior hand-knobs}

The first goal was to identify the functional networks obtained by seeding two precentral sectors showing differences in cortical excitability. The results from the seed-to-voxel second level analysis using the anterior and posterior hand-knob seeds identified in the two hemispheres are displayed in Figure 2 (only suprathreshold voxels are shown, $p<0.05$, FWE-corrected at the cluster level).

Defining the left and right anterior hand-knob sites as seeds produced a cluster of correlated resting-state BOLD activity in both hemispheres. Specifically, the functional network included the primary sensory and motor regions, the premotor areas of the caudal-most part of the superior, middle and inferior frontal gyri. These networks also involved, in both hemispheres, a region corresponding to the supplementary motor area (SMA), encompassing medially the anterior and posterior cingulate cortex and laterally to the central opercular cortex and the secondary somatosensory cortex, anterior supramarginal gyrus and superior parietal lobule. Finally, only the left anterior hand-knob emerged to be functional coupled with posterior and middle insula and the lateral occipitotemporal cortex(LOTC). Summarizing, the anterior hand-knob resulted to be functionally coupled with a bilateral network including frontoparietal cortical regions, the lateral occipitotemporal cortex and insular components.

We computed the resting state functional network of the left and right posterior hand-knob where HF-DES corresponding to the most excitable cortical sites. The functional networks obtained ( $p<0.05$, FWE-corrected at the cluster level; Fig. $2 C$, $D)$ were very similar to those obtained by seeding the anterior sectors (Fig. 2A,B), but some relevant differences also emerged. Specifically, first unlike the posterior hand-knob sector, the anterior sector lacks connections with the ventral-most portion of primary motor and sensory areas. Second, the anterior handknob functional network extends more anteriorly and posteriorly compared with the posterior hand-knob functional network. Third, while the lateral occipitotemporal cortex component is consistently present in the anterior hand-knob functional networks (Fig. $2 A, B$ ), it is rarely observed (Fig. $2 D$, right hemisphere) in the posterior hand-knob functional network.

Finally, it is noticeable that regardless of the seed, there was also a considerable FC between the four seeds.

\section{Comparison of FC of anterior and posterior hand-knob}

Given the great similarities in the FC of the anterior and posterior hand-knob sectors, to better evaluate significant differences in functionally coupling of the hand-knobs located within the same hemisphere, two-paired $t$ tests were conducted (i.e., $4 \times 2$ paired $t$ test: left anterior hand-knob $>$ left posterior hand-knob, left posterior hand-knob $>$ left anterior hand-knob, right anterior hand-knob $>$ right posterior hand-knob, and right posterior hand-knob $>$ right anterior hand-knob).

The results show that, bilaterally, main differences were found in frontal and parietal lobe (Fig. 3). The left and right anterior hand-knob resulted more strongly functionally coupled, compared with the ipsilateral posterior hand-knob, with the left and right precentral gyri, with the superior frontal gyri, with the SMA and with the central opercular cortex. It is noteworthy that the precentral sector whose spontaneous activity resulted to be more strongly connected with anterior hand-knob was composed, mainly in the comparison between the seeds located within the left hemisphere (Fig. $3 A$ ), by two different clusters one covering the ventral and the other lying on the dorsal portion of the premotor cortex.

Differences were also found in the parietal cortex. Bilaterally, the anterior hand-knobs, compared with the posteriors, were more functionally connected with the superior parietal lobe (SPL), with the anterior supramarginal gyrus, mainly in the comparison of the left hand-knobs seeds (Fig. 3A), and to the secondary somatosensory cortex as well as anterior intraparietal area (AIP). Finally, the anterior subdivision of the hand-knob was more strongly connected with the left and right insular cortex and the right lateral occipitotemporal cortex (Fig. $3 A$ ).

It is noteworthy that, the parietal and frontal regions with stronger functional coupling with the anterior hand-knob were smaller in the right hemisphere than in the left hemisphere (Fig. $3 B$ ). Furthermore, the paired $t$ test performed to display the cortical sectors more functionally coupled with the right anterior hand-knob, relative to the ipsilateral posterior one, failed in finding the insular and the lateral occipitotemporal cortex components (Fig. $3 B$ ), conversely highlighted in the other hemisphere (Fig. $3 A$ ).

Finally, the two paired $t$ test aimed at disclosing, within the same hemisphere, the cortical regions more functionally connected with the posterior hand-knob relative to the anterior one (left posterior hand-knob > left anterior hand-knob and right posterior hand-knob $>$ right anterior hand-knob) failed to find any significant voxel.

\section{Tractography}

We performed virtual dissections of the tracts extending from the hand-knob region using diffusion imaging to disclose whether the physiological differences highlighted by DES were reflected in different patterns of structural connections of the posterior and anterior hand-knob sectors. Using an average template of 1021 healthy subjects (HCP), U-fibers were traced 


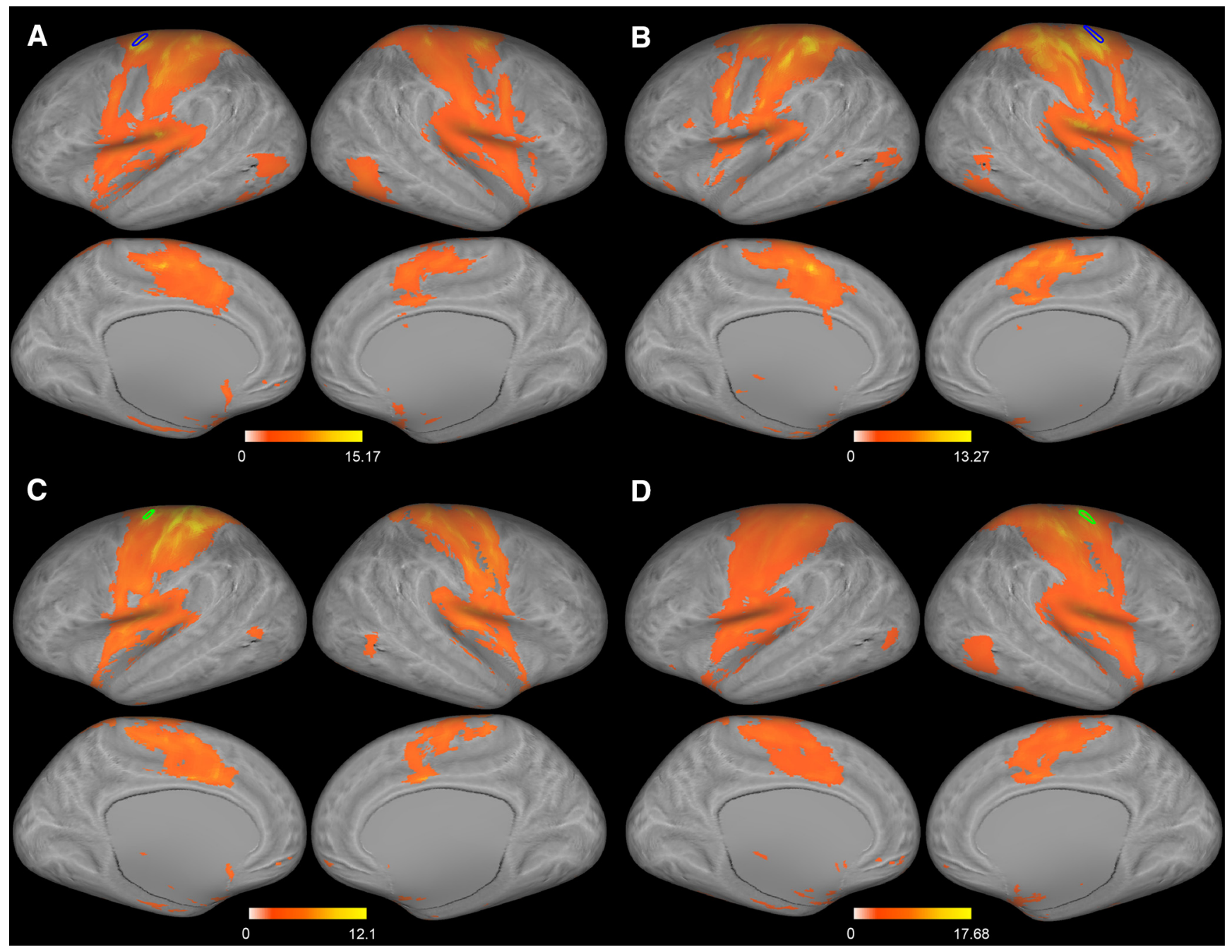

Figure 2. FC maps of the anterior (blue) and posterior (green) hand-knob identified intraoperatively. FC maps of the of left $(\boldsymbol{A})$ and right $(\boldsymbol{B})$ anterior hand-knob and of the left $(\boldsymbol{C})$ and right (D) posterior hand-knob.

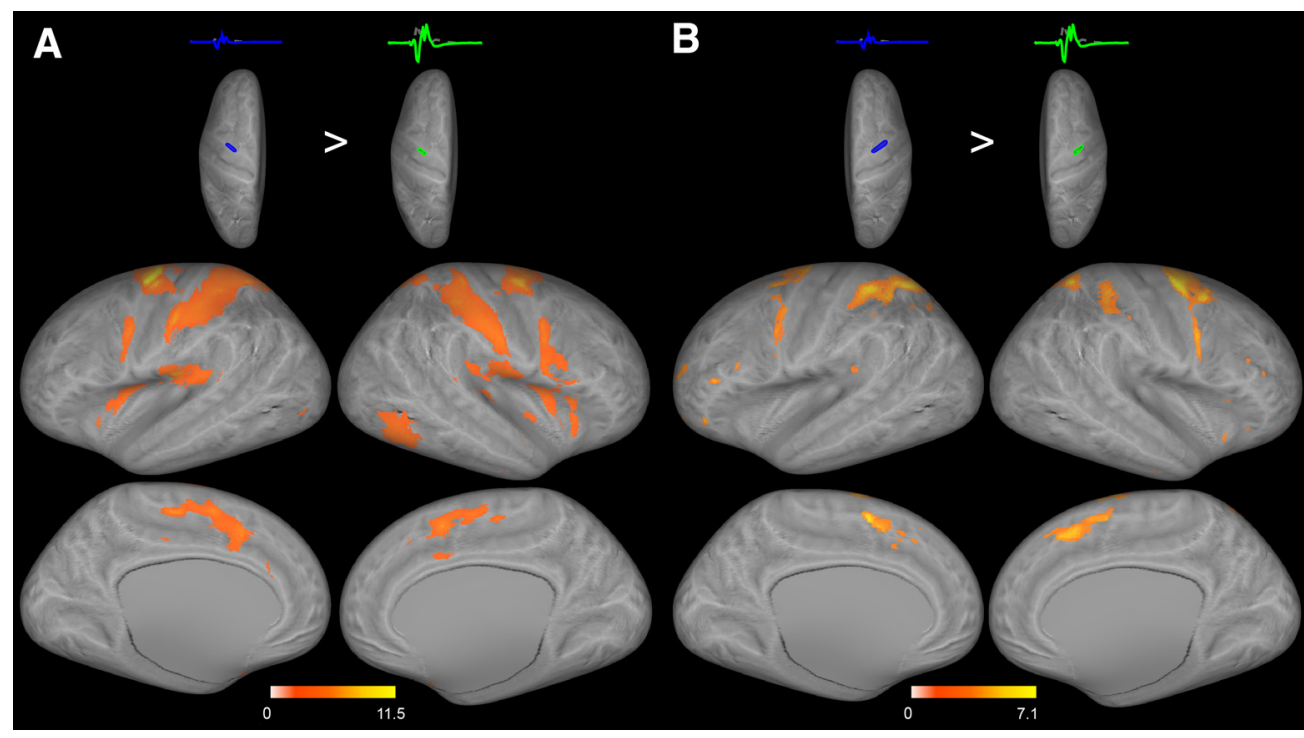

Figure 3. Comparison of $\mathrm{FC}$ of ipsilateral anterior and posterior hand-knob sectors of the left and right hemisphere (two paired $t$ tests height threshold of $<0.001 p$ uncorrected, cluster threshold $p<0.05 p$-FWE cluster-corrected). $\boldsymbol{A}$, $\mathrm{FC}$ of the left anterior hand-knob sector was stronger than $\mathrm{FC}$ of left posterior sector bilaterally in the precentral gyri, the superior frontal gyri, with SMA and with the central opercular cortex, with the SPL, with the anterior supramarginal gyrus, the secondary somatosensory cortex, AIP, LOC, and LOTC. B, FC of the right anterior handknob sector was stronger than that of the posterior one sector in a similar set of parietofrontal areas. 


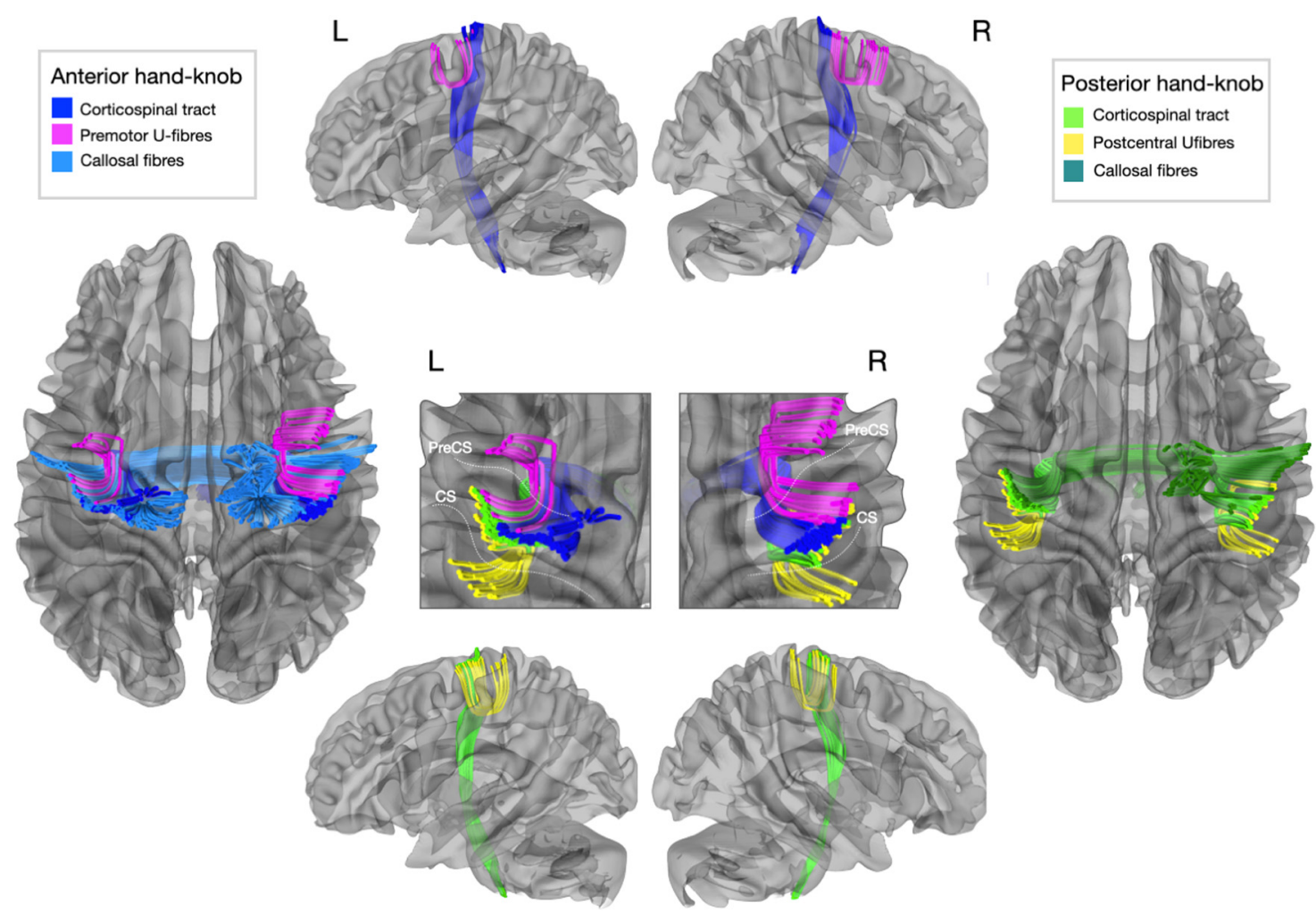

Figure 4. White matter connections of the anterior and posterior sectors. Both sectors contain corticofugal and callosal projections; however, the anterior sector is linked to connections with adjacent premotor regions, whereas the posterior sector is linked to connections with the postcentral gyrus.

between the left and right anterior hand-knob sectors and adjacent premotor cortical regions (Fig. 4). Additionally, in both hemispheres, tracts were identified connecting the posterior hand-knob and the postcentral gyrus. Corticofugal projections (CST) extended equally into both the posterior and anterior sectors of the precentral gyrus (Fig. 4). In addition, the two sectors showed strong connectivity with the contralateral hemisphere through callosal fibers (Fig. 4). Overall, these results suggest that the two sectors are characterized by distinct short frontal intralobar and parietofrontal connections.

\section{Discussion}

The main aim of this study was to infer the FC and SC, in healthy subjects, of two hand-knob sectors distinguished by different excitability, identified in tumor patients undergoing intraoperative brain-mapping. To this aim, the analysis of the connectivity of the cortical hand-knob seeds was conducted on healthy brains provided by the WU-Minn HCP consortium (Van Essen et al., 2013).

The novelty of this study lies in the method used to identify the seeds. The intraoperative electrophysiological brain-mapping, besides describing the causal relationship between DES and muscle activity, overcomes the spatial limitation of the indirect neuroimaging techniques, allowing an investigation of the cortex with higher resolution. This has identified two sectors within the precentral hand-knob, anterior and posterior, with different electrophysiological characteristics similar to what is observed in the monkey. The study of connectivity based on functional seeds allows to verify if, despite electrophysiological differences, the connectivity of the hand-knob sectors is similar.

Using rsfMRI, we found that the anterior and posterior handknob of both hemispheres show similar FC patterns, being both functionally coupled with precentral and postcentral gyri, the SMA, the secondary somatosensory area, the superior parietal cortex, the inferior parietal cortex, and the posterior and middle insula. However, some differences in the pattern of FC of these sectors emerged either in the relative extension of correlated cortical regions, or in the "preferential" functional coupling of some regions. A distinguishing feature of the functional networks of the two hand-knobs was represented by their FC within the central sulcus. While the anterior sector was functionally coupled only with the dorsal-most part of the central sulcus containing the hand representation, the posterior sector was functionally connected with the cortex along its entire dorsoventral extent. In line with other resting-state studies (Biswal et al., 1995; Power et al., 2011 the posterior hand-knob forms a unique network with M1 despite the somatotopic organization of M1 (Penfield and Boldrey, 1937; Meier et al., 2008). It has been demonstrated that M1 subregions are functionally correlated with contralateral regions occupying a similar spatial location (van den Heuvel and Hulshoff Pol, 2010), and that in primary motor and somatosensory areas, variations in myelin content overlap with intrinsic FC borders (Long et al., 2014; Kuehn et al., 2017). Therefore, although both hand-knob sectors host hand representation, only the anterior one forms a somatotopically organized resting-state network.

Although the differences in cortical excitability exhibited by the two hand-knob sectors suggest that different structural patterns of corticospinal connectivity exist, the absence of neuroanatomical data prevents validation. Furthermore, clinical constraints restrain us from exploring the excitability of the human M1 buried on the bank of the central sulcus. However, it is reasonable to hypothesize that stimulation of the posterior handknob sector may activate cortico-motoneuronal cells hosted in the central sulcus (Geyer et al., 1996), unlike stimulations 
delivered further away from the central sulcus that require a higher intensity to excite other or possibly the same neurons. Altogether these results are more in line with the hypothesis that the posterior hand-knob is located in M1 convexity, while the anterior hand-knob may represent a transition region from M1 toward the PMd. Interestingly, this hypothesis is supported by the cross-comparison results between their functional networks. Despite the widespread FC shared by the two sectors, their comparison shows that the anterior sector is strongly functionally coupled bilaterally with the precentral gyrus, the superior frontal gyrus, the SMA and the central opercular cortex. The parietal regions more strongly coupled with the anterior hand-knob were the SPL, the anterior supramarginal gyrus, hAIP and the secondary somatosensory cortex. The preferential connectivity of these regions with the anterior hand-knob confirms the evidence that rsfMRI is the result of the coactivation of regions embedded into the same functional network (Corbetta and Shulman, 2002; Smith et al., 2009). Indeed, imaging studies showed that cortical regions situated both in the precentral gyrus, the inferior parietal lobule and the inferior parietal sulcus play a role in the execution of object-oriented hand actions (Binkofski et al., 1999; Ehrsson et al., 2000; Culham et al., 2003). Moreover, TMS applied to both PMv and PMd (Davare et al., 2006, 2008, 2009) and to the SPL (Glover et al., 2005) impairs the execution of reach-to-grasp actions. Furthermore, the supramarginal gyrus is activated during the execution (Grèzes and Decety, 2001) of hand-object interactions and TMS-induced lesions of this region impairs planning of sequential goal-directed hand actions (Tunik et al., 2008). The role of SPL and premotor cortex in hand movement control has been confirmed by more invasive investigations (Desmurget et al., 2018; Fornia et al., 2020), where DES of these sectors led to the inhibition/impairment of hand movements. It is noteworthy that the two precentral sectors where stimulation impairs correct execution of hand-object manipulation (Fornia et al., 2020) emerge as more functionally connected with the anterior hand-knob.

A further difference in FC of the two hand-knob sectors was the presence of more consistent connectivity between the anterior sector and the occipitotemporal regions lateral occipital complex (LOC) and lateral occipitotemporal cortex (LOTC). The LOC is involved in perception object shape and processing volumetric features (Amedi et al., 2001, 2002; Moore and Engel, 2001). This region also plays a role in haptic coding of objects (Reed et al., 2004), tactile object recognition (Tal and Amedi, 2009), and hand action planning (Majdandzić et al., 2007). Interestingly, LOC object representation is based on actionrelated properties (Peelen et al., 2013). Furthermore, the LOTC is involved in visual processing related to the hand (Bracci et al., 2010) and for graspable objects including tools and seems to have a role in bridging object perception-related and parietal action-related representations (Bracci et al., 2016). In sum, the anterior hand-knob may be more embedded in circuits involved in sensorimotor transformations for grasping than the posterior sector, for which visual and somatosensory coding of the object's physical properties leads to the activation of distal movement representations adequate for hand-object interactions. Moreover, the FC of this sector with occipitotemporal sectors could provide it with visual information on object and actions for planning and online control of hand-object interactions.

In a previous study (Simone et al., 2020), we described the FC of a sector of the PMd where DES led to an increase in motor unit recruitment in hand muscles during execution of a hand manipulation task (recruitment sector). It is noteworthy that although the recruitment sector and the anterior hand-knob are quite distant, they show a similar FC pattern. Therefore, the anterior hand-knob, relative to the posterior one, is more functionally coupled with the cortical sectors involved in sensory-motor processes, thus showing a pattern of FC intermediate between that of the posterior hand-knob and those of the precentral sectors.

Although the exact relationship between rsfMRI and anatomic connectivity is still a matter of debate, it is largely accepted that FC is constrained by anatomic connectivity (Honey et al., 2009; Deco and Corbetta, 2011; Behrens and Sporns, 2012; Howells et al., 2020). We previously reported some differences in SC between the hand-knob sectors using tractography dissections performed in three tumor patients (Viganò et al., 2019). While the corticofugal projections extended toward both posterior and anterior hand-knob sectors, the superficial white matter (SWM) U-shaped tracts connecting dorsal premotor regions with the precentral gyrus predominantly terminated within the anterior hand-knob, while the tracts connecting the precentral gyrus with the postcentral gyrus terminated in the posterior hand-knob. These differences were also identified when examining the connectivity of each sector using a population-average template of 1021 healthy subjects provided by the HCP (Van Essen et al., 2013). The differential patterns of short-range corticocortical U-shaped connections along the anteroposterior direction may support the hypothesis that the two sectors play different roles in hand motor control (Catani et al., 2012; Thompson et al., 2017). Indeed, the direct connection between M1 and the somatosensory cortex, intersecting the posterior hand-knob, likely plays a crucial role in providing somatosensory information for skillful hand motor ability (Thompson et al., 2017). Conversely, the fibers connecting dorsal premotor regions with the precentral anterior hand-knob sector support its role in sensorimotor transformation. Tractography provides, however, an estimation of the projections of white matter pathways, and is subject to methodological limitations. First, it is well known that PMd and M1 are directly and reciprocally connected in the macaque (Marconi et al., 2001; Dum and Strick, 2005), yet tractography at this resolution is unable to track intragyral fibers running within the hand-knob region. More complex fiber orientations than adjacent gyrus-to-gyrus connectivity has been described in different studies (Shinohara et al., 2020). As our study has used a population-average template, this will have omitted more heterogeneous fibers, which may include these fibers traversing through the gyrus. Studies using postmortem methods and ironcontrast imaging may be able to better identify the connectivity of these regions (Kirilina et al., 2020).

The functional definition of the two hand-knob sectors, within a large cohort of patients, show they potentially play different roles in motor control suggesting that the human handknob is an anatomo-functional heterogeneous region organized along a motor-cognitive gradient.

\section{References}

Amedi A, Malach R, Hendler T, Peled S, Zohary E (2001) Visuo-haptic object-related activation in the ventral visual pathway. Nat Neurosci 4:324-330.

Amedi A, Jacobson G, Hendler T, Malach R, Zohary E (2002) Convergence of visual and tactile shape processing in the human lateral occipital complex. Cereb Cortex 12:1202-1212.

Behrens TE, Sporns O (2012) Human connectomics. Curr Opin Neurobiol 22:144-153.

Bello L, Riva M, Fava E, Ferpozzi V, Castellano A, Raneri F, Pessina F, Bizzi A, Falini A, Cerri G (2014) Tailoring neurophysiological strategies with 
clinical context enhances resection and safety and expands indications in gliomas involving motor pathways. Neuro Oncol 16:1110-1128.

Binkofski F, Buccino G, Stephan KM, Rizzolatti G, Seitz RJ, Freund HJ (1999) A parieto-premotor network for object manipulation: evidence from neuroimaging. Exp Brain Res 128:210-213.

Biswal B, Yetkin FZ, Haughton VM, Hyde JS (1995) Functional connectivity in the motor cortex of resting human brain using echo-planar MRI. Magn Reson Med 34:537-541.

Bracci S, Ietswaart M, Peelen MV, Cavina-Pratesi C (2010) Dissociable neural responses to hands and non-hand body parts in human left extrastriate visual cortex. J Neurophysiol 103:3389-3397.

Bracci S, Cavina-Pratesi C, Connolly JD, Ietswaart M (2016) Representational content of occipitotemporal and parietal tool areas. Neuropsychologia 84:81-88.

Catani M, Dell'acqua F, Vergani F, Malik F, Hodge H, Roy P, Valabregue R, Thiebaut de Schotten M (2012) Short frontal lobe connections of the human brain. Cortex 48:273-291.

Corbetta M, Shulman GL (2002) Control of goal-directed and stimulusdriven attention in the brain. Nat Rev Neurosci 3:201-215.

Crone NE, Miglioretti DL, Gordon B, Lesser RP (1998) Functional mapping of human sensorimotor cortex with electrocorticographic spectral analysis. II. Event-related synchronization in the gamma band. Brain 121:2301-2315.

Culham JC, Danckert SL, DeSouza JFX, Gati JS, Menon RS, Goodale MA (2003) Visually guided grasping produces fMRI activation in dorsal but not ventral stream brain areas. Exp Brain Res 153:180-189.

Davare M, Andres M, Cosnard G, Thonnard JL, Olivier E (2006) Dissociating the role of ventral and dorsal premotor cortex in precision grasping. J Neurosci 26:2260-2268.

Davare M, Lemon R, Olivier E (2008) Selective modulation of interactions between ventral premotor cortex and primary motor cortex during precision grasping in humans. J Physiol 586:2735-2742.

Davare M, Montague K, Olivier E, Rothwell JC, Lemon RN (2009) Ventral premotor to primary motor cortical interactions during object-driven grasp in humans. Cortex 45:1050-1057.

Deco G, Corbetta M (2011) The dynamical balance of the brain at rest. The Neuroscientist 17:107-123.

Desmurget M, Richard N, Beuriat PA, Szathmari A, Mottolese C, Duhamel JR, Sirigu A (2018) Selective inhibition of volitional hand movements after stimulation of the dorsoposterior parietal cortex in humans. Curr Biol 28:3303-3309.e3.

Dum RP, Strick PL (2005) Frontal lobe inputs to the digit representations of the motor areas on the lateral surface of the hemisphere. J Neurosci 25:1375-1386.

Ehrsson HH, Fagergren A, Jonsson T, Westling G, Johansson RS, Forssberg $\mathrm{H}$ (2000) Cortical activity in precision- versus power-grip tasks: an fMRI study. J Neurophysiol 83:528-536.

Fischl B (2012) FreeSurfer. Neuroimage 62:774-781.

Fornia L, Ferpozzi V, Montagna M, Rossi M, Riva M, Pessina F, Martinelli Boneschi F, Borroni P, Lemon RN, Bello L, Cerri G (2018) Functional characterization of the left ventrolateral premotor cortex in humans: a direct electrophysiological approach. Cereb Cortex 28:167-183.

Fornia L, Rossi M, Rabuffetti M, Leonetti A, Puglisi G, Viganò L, Simone L, Howells H, Bellacicca A, Bello L, Cerri G (2020) Direct electrical stimulation of premotor areas: different effects on hand muscle activity during object manipulation. Cereb Cortex 30:391-405.

Friston KJ, Holmes AP, Poline JB, Grasby PJ, Williams SC, Frackowiak RS, Turner R (1995) Analysis of fMRI time-series revisited. Neuroimage 2:45-53.

Geyer S, Ledberg A, Schleicher A, Kinomura S, Schormann T, Bürgel U, Klingberg T, Larsson J, Zilles K, Roland PE (1996) Two different areas within the primary motor cortex of man. Nature 382:805-807.

Glasser MF, Sotiropoulos SN, Wilson JA, Coalson TS, Fischl B, Andersson JL, Xu J, Jbabdi S, Webster M, Polimeni JR, Van Essen DC, Jenkinson M; WU-Minn HCP Consortium (2013) The minimal preprocessing pipelines for the Human Connectome Project. Neuroimage 80:105-124.

Glover S, Miall RC, Rushworth MF (2005) Parietal rTMS disrupts the initiation but not the execution of on-line adjustments to a perturbation of object size. J Cogn Neurosci 17:124-136.

Grèzes J, Decety J (2001) Functional anatomy of execution, mental simulation, observation, and verb generation of actions: a meta-analysis. Hum Brain Mapp 12:1-19.
Honey CJ, Sporns O, Cammoun L, Gigandet X, Thiran JP, Meuli R, Hagmann P (2009) Predicting human resting-state functional connectivity from structural connectivity. Proc Natl Acad Sci USA 106:2035-2040.

Howells H, Simone L, Borra E, Fornia L, Cerri G, Luppino G (2020) Reproducing macaque lateral grasping and oculomotor networks using resting state functional connectivity and diffusion tractography. Brain Struct Funct 225:2533-2551.

Kirilina E, Helbling S, Morawski M, Pine K, Reimann K, Jankuhn S, Dinse J, Deistung A, Reichenbach JR, Trampel R, Geyer S, Müller L, Jakubowski N, Arendt T, Bazin PL, Weiskopf N (2020) Superficial white matter imaging: contrast mechanisms and whole-brain in vivo mapping. Sci Adv 6: eaaz9281.

Kuehn E, Dinse J, Jakobsen E, Long X, Schäfer A, Bazin PL, Villringer A, Sereno MI, Margulies DS (2017) Body topography parcellates human sensory and motor cortex. Cereb Cortex 27:3790-3805.

Long X, Goltz D, Margulies DS, Nierhaus T, Villringer A (2014) Functional connectivity-based parcellation of the human sensorimotor cortex. Eur J Neurosci 39:1332-1342.

Majdandzić J, Gro MJ, van Schie HT, Verhagen L, Toni I, Bekkering H (2007) The role of immediate and final goals in action planning: an fMRI study. Neuroimage 37:589-598.

Maranesi M, Rodà F, Bonini L, Rozzi S, Ferrari PF, Fogassi L, Coudé G (2012) Anatomo-functional organization of the ventral primary motor and premotor cortex in the macaque monkey. Eur J Neurosci 36:33763387.

Marconi B, Genovesio A, Battaglia-Mayer A, Ferraina S, Squatrito S, Molinari M, Lacquaniti F, Caminiti R (2001) Eye-hand coordination during reaching. I. Anatomical relationships between parietal and frontal cortex. Cereb Cortex 11:513-527.

Meier JD, Aflalo TN, Kastner S, Graziano MS (2008) Complex organization of human primary motor cortex: a high-resolution fMRI study. J Neurophysiol 100:1800-1812.

Moore C, Engel SA (2001) Neural response to perception of volume in the lateral occipital complex. Neuron 29:277-286.

Peelen MV, Bracci S, Lu X, He C, Caramazza A, Bi Y (2013) Tool selectivity in left occipitotemporal cortex develops without vision. J Cogn Neurosci 25:1225-1234.

Penfield W, Boldrey E (1937) Somatic motor and sensory representation in the cerebral cortex of man as studied by electrical stimulation. Brain 60:389-443.

Porter R, Lemon RN (1993) Corticospinal function and voluntary movement, p 428. Oxford: Clarendon Press.

Power JD, Cohen AL, Nelson SM, Wig GS, Barnes KA, Church JA, Vogel AC, Laumann TO, Miezin FM, Schlaggar BL, Petersen SE (2011) Functional network organization of the human brain. Neuron 72:665678.

Puglisi G, Sciortino T, Rossi M, Leonetti A, Fornia L, Conti NM, Casarotti A, Pessina F, Riva M, Cerri G, Bello L (2018) Preserving executive functions in non- dominant frontal lobe glioma surgery: an intraoperative tool. J Neurosurg 28:1-7.

Puglisi G, Howells H, Sciortino T, Leonetti A, Rossi M, Conti NM, Gay LG, Fornia L, Bellacicca A, Viganò L, Simone L, Catani M, Cerri G, Bello L (2019) Frontal pathways in cognitive control: evidence from intraoperative stimulation and tractography. Brain 142:2451-2465.

Rathelot JA, Strick PL (2009) Subdivisions of primary motor cortex based on cortico-motoneuronal cells. Proc Natl Acad Sci USA 106:918-923.

Rech F, Herbet G, Gaudeau Y, Mézières S, Moureau JM, Moritz-Gasser S, Duffau H (2019) A probabilistic map of negative motor areas of the upper limb and face: a brain stimulation study. Brain 142:952-965.

Reed CL, Shoham S, Halgren E (2004) Neural substrates of tactile object recognition: an fMRI study. Hum Brain Mapp 21:236-246.

Rizzolatti G, Camarda R, Fogassi L, Gentilucci M, Luppino G, Matelli M (1988) Functional organization of inferior area 6 in the macaque monkey: II. Area F5 and the control of distal movements. Exp Brain Res 71:491507.

Rossi M, Fornia L, Puglisi G, Leonetti A, Zuccon G, Fava E, Milani D, Casarotti A, Riva M, Pessina F, Cerri G, Bello L (2018) Assessment of the praxis circuit in glioma surgery to reduce the incidence of postoperative and long-term apraxia: a new intraoperative test. J Neurosurg 23:1-11.

Scott DW (2015) Multivariate density estimation: theory, practice, and visualization, Ed 2. Hoboken: Wiley. 
Shinohara H, Liu X, Nakajima R, Kinoshita M, Ozaki N, Hori O, Nakada M (2020) Pyramid-shape crossings and intercrossing fibers are key elements for construction of the neural network in the superficial white matter of the human cerebrum. Cereb Cortex 30:5218-5228.

Silverman BW (1986) Density estimation for statistics and data analysis. Boca Raton: Chapman and Hall/CRC.

Simone L, Fornia L, Viganò L, Sambataro F, Rossi M, Leonetti A, Puglisi G, Howells H, Bellacicca A, Bello L, Cerri G (2020) Large scale networks for human hand-object interaction: functionally distinct roles for two premotor regions identified intraoperatively. Neuroimage 204:116215.

Smith SM, Fox PT, Miller KL, Glahn DC, Fox PM, Mackay CE, Filippini N, Watkins KE, Toro R, Laird AR, Beckmann CF (2009) Correspondence of the brain's functional architecture during activation and rest. Proc Natl Acad Sci USA 106:13040-13045.

Smith SM, Beckmann CF, Andersson J, Auerbach EJ, Bijsterbosch J, Douaud G, Duff E, Feinberg DA, Griffanti L, Harms MP, Kelly M, Laumann T, Miller KL, Moeller S, Petersen S, Power J, Salimi-Khorshidi G, Snyder AZ, Vu AT, Woolrich MW, et al. (2013) Resting state fMRI in the human connectome project. Neuroimage 80:144-168.

Spinnler H, Tognoni G (1987) [Italian standardization of neuropsychological tests.] Ital J Neurol Sci 8:1-120.

Strick PL, Preston JB (1982) Two representations of the hand in area 4 of a primate. II. Somatosensory input organization. J Neurophysiol 48:150159.e9.

Tal N, Amedi A (2009) Multisensory visual-tactile object related network in humans: insights gained using a novel crossmodal adaptation approach. Exp Brain Res 198:165-182.

Thompson A, Murphy D, Dell'Acqua F, Ecker C, McAlonan G, Howells H, Baron-Cohen S, Lai MC, Lombardo MV, MRC AIMS Consortium, Catani M (2017) Impaired communication between the motor and somatosensory homunculus is associated with poor manual dexterity in autism spectrum disorder. Biol Psychiatry 81:211-219.
Tunik E, Lo OY, Adamovich SV (2008) Transcranial magnetic stimulation to the frontal operculum and supramarginal gyrus disrupts planning of outcome-based hand-object interactions. J Neurosci 28:14422-14427.

Uğurbil K, Xu J, Auerbach EJ, Moeller S, Vu AT, Duarte-Carvajalino JM, Lenglet C, Wu X, Schmitter S, Van de Moortele PF, Strupp J, Sapiro G, De Martino F, Wang D, Harel N, Garwood M, Chen L, Feinberg DA, Smith SM, Miller KL, et al. (2013) Pushing spatial and temporal resolution for functional and diffusion MRI in the human connectome project. Neuroimage 80:80-104.

van den Heuvel MP, Hulshoff Pol HE (2010) Specific somatotopic organization of functional connections of the primary motor network during resting state. Hum Brain Mapp 31:631-644.

Van Essen DC, Smith SM, Barch DM, Behrens TE, Yacoub E, Uğurbil K; WU-Minn HCP Consortium (2013) The WU-Minn Human Connectome Project: an overview. Neuroimage 80:62-79.

Viganò L, Fornia L, Rossi M, Howells H, Leonetti A, Puglisi G, Conti Nibali M, Bellacicca A, Grimaldi M, Bello L, Cerri G (2019) Anatomo-functional characterisation of the human "hand-knob": a direct electrophysiological study. Cortex 113:239-254.

Yeh FC, Tseng WY (2011) NTU-90: a high angular resolution brain atlas constructed by q-space diffeomorphic reconstruction. Neuroimage 58:91-99.

Yeh FC, Wedeen VJ, Tseng WY (2010) Generalized q-sampling imaging. IEEE Trans Med Imaging 29:1626-1635.

Yeh FC, Panesar S, Fernandes D, Meola A, Yoshino M, Fernandez-Miranda JC, Vettel JM, Verstynen T (2018) Population-averaged atlas of the macroscale human structural connectome and its network topology. Neuroimage 178:57-68.

Yousry TA, Schmid UD, Alkadhi H, Schmidt D, Peraud A, Buettner A, Winkler P (1997) Localization of the motor hand area to a knob on the precentral gyrus. A new landmark. Brain 120:141-157. 